\title{
La educación de las niñas en países en desarrollo: aspectos destacados de la investigación del Population Council
}

Cynthia B. Lloyd

Population Council

Follow this and additional works at: https://knowledgecommons.popcouncil.org/departments_sbsr-pgy

Part of the Demography, Population, and Ecology Commons, Education Commons, Family, Life Course, and Society Commons, Gender and Sexuality Commons, and the International Public Health Commons How does access to this work benefit you? Let us know!

\section{Recommended Citation}

Lloyd, Cynthia B. 2011. "La educación de las niñas en países en desarrollo: aspectos destacados de la investigación del Population Council," Promoción de transiciones a la vida adulta sanas, seguras y productivas Resumen no. 24. New York: Population Council. 


\section{La educación de las niñas en países en desarrollo: aspectos destacados de la investigación del Population Council}

\section{Preparado por Cynthia B. Lloyd}

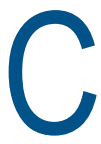

ada vez más niñas asisten a la escuela en la adolescencia. Por esta razón, es clara la importancia de las experiencias escolares y la calidad de las escuelas para la salud reproductiva y sexual de las adolescentes, así como para una exitosa transición a la edad adulta. La investigación del Population Council sobre educación busca comprender mejor los patrones y las tendencias en la educación para niñas y más particularmente la relación entre las experiencias escolares, la calidad de las escuelas y las diversas consecuencias para los adolescentes, lo que incluye: asistencia a la escuela y logros en ella; aprovechamiento del tiempo y rol laboral; salud reproductiva y sexual; actitud frente a cuestiones de género; ciudadanía y participación en la comunidad; transición al matrimonio y la crianza de los hijos.

Los aspectos destacados de este programa de investigación se resumen a continuación en cinco amplios subtemas: (1) demografía de la educación; (2) asistencia a la escuela y sus beneficios para las niñas; (3) experiencias reproductivas y sexuales y evolución en la escuela; (4) igualdad de género, actitud de los maestros y calidad de las escuelas; y 5) participación de las adolescentes en el sector de la educación no formal. La investigación fue comparativa y exhaustiva en los países seleccionados, incluidos Bangladesh, Egipto, Guatemala, Kenia, Pakistán, Senegal y Sudáfrica. Se realizó en paralelo con el desarrollo y la evaluación de programas destinados a abordar las necesidades de las niñas que no asisten a la escuela y de otros grupos de niñas marginales. Estos programas apuntan a apoyar a las niñas que asisten a la escuela, ayudarlas a que vuelvan en caso de que la hayan abandonado y proporcionar espacios seguros donde puedan aprender diversas habilidades para la vida cotidiana.

La educación universal contribuye a la disminución de la fertilidad, pero a pesar de eso, las diferencias de género persisten En África subsahariana, están desapareciendo las diferencias de género en lo que respecta a educación. No obstante, los niveles de participación están muy por debajo de lo que se requiere para lograr la enseñanza primaria universal para 2015 (segundo Objetivo de Desarrollo del Milenio). Se han utilizado datos de la encuesta nacional de hogares sobre participación y logros en educación, en 24 países de África subsahariana, para obtener

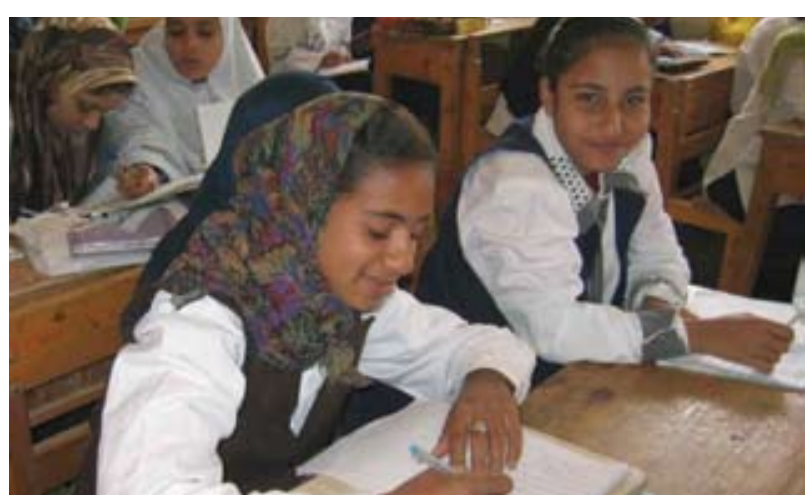

un cálculo alternativo de los niveles y tendencias en educación, con un enfoque particular en las diferencias de género (Hewett y Lloyd 2005). El análisis de datos esclarece algunas de las inclinaciones inherentes a los datos basados en las escuelas presentados por la UNESCO, en particular, una diferencia de género potencialmente exagerada.

No obstante, las diferencias de género persisten en grupos vulnerables (poblaciones rurales, minorías étnicas u otras poblaciones excluidas). Un análisis en profundidad, que se realizó en Guatemala (Hallman et al. 2007), Pakistán (Lloyd et al. 2007) y Senegal (Montgomery y Hewett 2006), documenta las grandes diferencias de género en lo que respecta a participación y logros en educación que persisten en poblaciones rurales (geográficamente aisladas) y marginadas 0 excluidas por razones étnicas. Estos análisis demuestran las desventajas que sufren las niñas de estos grupos, en porcentajes que muchas veces se duplican, triplican y hasta cuadruplican.

La investigación comparativa en África (Lloyd, Kaufman y Hewett 2000) y la investigación exhaustiva en Pakistán (Sathar et al. 2003) demuestran la importancia de lograr una educación masiva para que disminuya la fertilidad. Todos los países subsaharianos que lograron una educación masiva, es decir, que un 75 por ciento de los jóvenes llega a cuarto año 0 a un año superior, comenzaron la transición de la fertilidad. En el área rural de Pakistán, el logro de la igualdad de género en el acceso a las escuelas estuvo fuertemente relacionado con la expresión de las mujeres de menos deseos de fertilidad y, por consiguiente, su predisposición a utilizar anticonceptivos para limitar el tamaño de la familia. 
El programa Ishraq en la zona rural de Alto Egipto: abrir el camino para el reingreso en la educación formal

En 2001, El Population Council y Save the Children, en sociedad con Caritas y el Centro de Actividades de Desarrollo y Población (CEDPA), diseñaron e implementaron un programa piloto, denominado Ishraq ("progresismo"), para abordar las necesidades de niñas de entre 13 y 15 años que no asisten a la escuela en varias aldeas rurales de la gobernación de Al Minya en Egipto. El programa tiene múltiples objetivos, que incluyen la promoción de la alfabetización, la enseñanza de habilidades para la vida cotidiana, el desarrollo de redes sociales, el fomento del liderazgo y la confianza en uno mismo a través de los deportes y la reintegración de los participantes en el sistema de educación formal. En 2009, Ishraq se extendió a todo Alto Egipto y, hasta la fecha, 1.800 niñas han participado en el programa. www.popcouncil.org/projects/ 40_IshraqSafeSpacesGirls.asp\#/Resources

\section{En el caso de las niñas, la asistencia a la escuela conduce a múltiple beneficios}

Ciertos beneficios para las niñas relacionados con la asistencia a la escuela parecen casi universales, independientemente del tipo o calidad de la escuela a la que asistan. Por ejemplo, una mayor igualdad de género entre los niños y niñas respecto del aprovechamiento del tiempo y la carga de trabajos mientras están inscriptos (Lloyd et al. (en preparación); Arends-Kuenning y Amin 2004; Lloyd y Grant 2005; Lloyd 2005), un menor riesgo de tener relaciones sexuales prematrimoniales, en especial por parte de las niñas (Lloyd 2005) y una disminución de matrimonios a edades tempranas, particularmente en África subsahariana (Mensch, Singh y Casterline 2005). Además, parece ser que las adolescentes que comienzan con la actividad sexual mientras asisten a la escuela tienden más a usar anticonceptivos que las adolescentes de su misma edad que ya no van a la escuela; así, corren un riesgo menor de tener un embarazo no deseado o de contraer una enfermedad de transmisión sexual, como el VIH/SIDA (Lloyd 2005).

No obstante, en sociedades como Bangladesh y Egipto, donde ciertas actitudes tradicionales hacia cuestiones de género siguen muy arraigadas, la mayor participación de las niñas en la educación no sirvió para cambiarlas (Mensch et al. 2003), como tampoco sirvió para mejorar las posibilidades de obtener un trabajo en el caso de Egipto (Amin y AlBassusi 2004), ni para retardar la edad en la que contraen matrimonio en el caso de Bangladesh (Mahmud y Amin 2006), pero sí estuvo relacionada con otros beneficios. Así, la mayor participación de las niñas en educación puede conferirles ciertos beneficios temporales durante la adolescencia, pero al menos algunos de los resultados beneficiosos pueden desaparecer cuando las jóvenes pasan a cumplir un papel tradicional adulto, en especial en sociedades con culturas locales que valoran la fuerte diferenciación de género.
Los riesgos de la salud reproductiva y sexual para las niñas constituyen una amenaza para su progreso en la escuela Cuando las niñas alcanzan la pubertad, corren ciertos riesgos, como ser sometidas a relaciones sexuales por la fuerza, embarazos no deseados, matrimonios a edades tempranas y $\mathrm{VIH}$, que pueden interferir con su progreso en la escuela o incluso forzarlas a abandonar. En particular, las relaciones prematrimoniales, incluidas las relaciones sexuales no consensuadas, pueden llevar al abandono de la escuela y siguen siendo un riesgo que las adolescentes tienen que poder manejar mientras asisten a la escuela. Los resultados de estudios en Sudáfrica (Hallman 2007) y en Burkina Faso, Ghana, Malawi y Uganda (Biddlecom et al. 2006) documentan que los riesgos de abandono son significativamente mayores para las niñas que han mantenido relaciones sexuales prematrimoniales. La relación entre la inscripción en las escuelas y la experiencia de las niñas con eventos reproductivos es más compleja hoy que en el pasado, y esto se debe a la mayor cantidad de adolescentes que asisten a la escuela y a la cantidad de tiempo durante la adolescencia que pasan en la escuela.

Un análisis comparativo con datos de varias encuestas en África francófona (Lloyd y Mensch 2008) demuestra que los riesgos de abandonar la escuela durante la adolescencia por razones que no sean el nacimiento de un hijo o el matrimonio, como una mala nota en un examen 0 limitaciones económicas, superan en gran medida los riesgos relacionados con dichos eventos demográficos. Además, los riesgos de abandonar la escuela por estas razones tradicionales han disminuido durante los últimos 20 años, a pesar de que hubo un aumento en el porcentaje de adolescentes que siguen yendo a la escuela después de haber alcanzado la pubertad y la prolongada exposición a los riesgos de quedar embarazada al salir de la escuela a una edad más avanzada. Esta relación evolutiva entre los eventos reproductivos y la educación requiere un análisis cuidadoso que permita garantizar que las políticas y los programas apoyen resultados positivos y protejan a las niñas de riesgos aparentes.

\section{Igualdad de género; la actitud de los maestros influye} enormemente en la calidad de las escuelas

Para comprender el efecto global de la educación de las niñas es importante medir otras cuestiones que van más allá de los elementos tradicionales relacionados con la calidad de las escuelas (instalaciones, prácticas pedagógicas, tiempo para aprender) y que están asociados con el aprendizaje de habilidades académicas básicas de alfabetización, cálculos y pensamiento crítico. Un enfoque más amplio sobre la calidad de las escuelas también incluye la salud, en especial la salud reproductiva, el desarrollo de actitudes y comportamientos que favorecen la igualdad de género, la confianza en uno mismo y habilidades para la toma de decisiones. El objetivo de la investigación del Population Council ha sido identificar tales factores y documentar sus consecuencias. Claramente, la actitud y el comportamiento de los maestros influyen enormemente en la calidad de las escuelas.

En lugares tan variados como Egipto, Kenia y Pakistán, la investigación del Population Council demostró que las niñas responden mejor a diversas dimensiones relacionadas con la calidad de las escuelas

2 - Visite www.popcouncil.org/publications/serialsbriefs/TABriefs.asp para obtener todos los resúmenes de la serie Promoting healthy, safe, and productive transitions to adulthood (Promoción de transiciones a la vida adulta sanas, seguras y productivas). 
que los niños. Especialmente en Kenia, ciertos aspectos del ambiente escolar, que incluyen los niveles de apoyo y el asesoramiento por parte de los maestros, las actitudes de los maestros hacia la importancia relativa de las matemáticas para las niñas y los niños, y experiencias de hostilidad y tratamiento desigual, tienden a influir más en el porcentaje de abandonos de las niñas que de los niños (Lloyd, Mensch y Clark 2000). En Egipto, donde se considera que las niñas se comportan bien y tienen facilidad para aprender, muchos de los factores que influyen en el abandono escolar de las niñas están relacionados con elementos más tradicionales de la calidad de las escuelas. El trato de los maestros, por ejemplo el hecho de que un maestro le diga a un estudiante que es un fracaso, es importante tanto para los niños como para las niñas, en términos de porcentajes de abandono (Lloyd et al. 2003). En el área rural de Pakistán, las decisiones de inscripción y permanencia responden a la calidad de las escuelas. En lo que respecta a inscripción, se descubrió que la cantidad de maestros que residen en la comunidad (algo fácilmente reconocible por los padres) influye muy particularmente en la inscripción de las niñas. En lo que respecta a permanencia, el hecho de asistir a una escuela pública en vez de a una privada (las escuelas públicas tienden a tener un nivel inferior en todos los aspectos de la calidad de las escuelas) aumenta significativamente las probabilidades de que las niñas abandonen la escuela (Lloyd et al. 2005. Lloyd et al. 2006). La falta de maestros, que es más frecuente en las escuelas públicas para niñas dado su bajo nivel de educación y su dependencia de maestras no locales, disminuye el acceso de las niñas a la escuela. Al momento de las visitas a las escuelas, el 25 por ciento de niñas inscriptas en la zona rural de Pakistán no contaba con la presencia de un maestro en la clase (Ghuman y Lloyd 2007).

Los porcentajes de inscripción y abandono no son las únicas consecuencias para los adolescentes que se ven afectados por la calidad de las escuelas. En Kenia, una escuela que se caracteriza por brindar una atmósfera neutra en cuanto al género parece disminuir el riesgo de que las niñas se involucren en relaciones sexuales prematrimoniales en comparación con una escuela que tiende a discriminar a las niñas (Mensch et al. 2001). En Sudáfrica, el bajo rendimiento de las niñas en la escuela se mide por la cantidad de repeticiones de año y el abandono está muy vinculado con la posibilidad de que queden embarazadas mientras asisten a la escuela (Grant y Hallman 2006). Una evaluación del programa de educación en habilidades para la vida cotidiana aplicado en todas las escuelas secundarias de Sudáfrica en 2005 descubrió efectos significativos sobre el conocimiento y las actitudes y el uso de preservativos, pero no arrojó diferencias en la edad de iniciación sexual ni una disminución en la cantidad de parejas, lo que indica que la calidad del programa no es suficiente para lograr todos los objetivos establecidos (Horizons Program 2004).

\section{Aún se desconocen los beneficios actuales y futuros de la participación de las adolescentes en los programas de la educación no formal}

Junto con el empuje para lograr la enseñanza universal durante los últimos 10 años, vino un aumento aparente en la cantidad de escuelas no gubernamentales, que por lo general son escuelas no formales (UNESCO
2007). Un reciente informe del Population Council, titulado: New Lessons: The Power of Educating Adolescent Girls, encargado por la Fundación de las Naciones Unidas y la Fundación Nike, indica que la educación no formal les abre el camino hacia la educación a niñas en desventaja y documenta muchos ejemplos de programas educativos no formales que actualmente les brindan una educación alternativa a las adolescentes (Lloyd y Young 2009). En forma irónica, la cantidad de inscripciones en escuelas no formales no aparece en los datos nacionales sobre inscripción en las escuelas, lo que minimiza la notoriedad de estos esfuerzos y la capacidad de contribuir a los objetivos de desarrollo. Además, aún no se conocen la cobertura y el impacto para las adolescentes en el corto plazo, en lo que respecta al avance en la escuela, y en el largo plazo, en lo que respecta a lo bien que las niñas se preparan para la adultez, el trabajo remunerado y la administración del hogar.

\section{Implicancia de la política}

Aunque, en muchos países, el porcentaje de inscripción en las escuelas para las niñas aumente y las diferencias de género disminuyan, las niñas en subpoblaciones vulnerables siguen bastante rezagadas y requieren especial apoyo para ser integradas en los sistemas de educación formal. Los programas educativos no formales complementarios, diseñados para lograr que los niños vuelvan a ingresar en el sistema de educación formal, se convertirán en una estrategia cada vez más importante y permitirán que las adolescentes avancen a la escuela secundaria formal. Las pruebas cada vez mayores de una pobre enseñanza en muchos países en desarrollo hacen que los encargados de formular políticas educativas reconozcan la importancia de invertir para lograr escuelas de mejor calidad. Los resultados de la investigación del Population Council sobre educación resaltaron la importancia de muchas dimensiones de la calidad de las escuelas que pueden ser fundamentales para el éxito en la edad adulta, en particular en el caso de las niñas, cuyo comportamiento y avance en la escuela parece ser más sensible al entorno educativo que el de los niños.

Además, New Lessons: The Power of Educating Adolescent Girls brinda un compendio de programas educativos para adolescentes que incluyen a las niñas y destaca la necesidad de obtener más información sobre los porcentajes sociales y económicos de niñas que se inscriben en el sector de la educación no formal, que cada vez crece más (Lloyd y Young 2009). A medida que los países intenten captar los beneficios de un mayor acceso a la educación por parte de sus jóvenes, se beneficiarán de las evaluaciones de estos aspectos de la educación y sus repercusiones para los resultados deseados.

\section{Referencias y publicaciones relacionadas}

Amin, S. y N.H. Al-Bassusi. 2004. "Education, wage work and marriage; Perspectives of Egyptian working women," Journal of Marriage and Family 66: 1287-1299.

Amin, S. (en preparación). "Bangladesh: achieving universal primary education at the end of the 20th century," en A. Gupta (ed.), Schooling Around the World: South Asia. New York: Routledge.

Arends-Kuenning, M. y S. Amin. 2001. "Women's capabilities and the right to education in Bangladesh," International Journal of Politics, Culture and Society 15(1): 125-142. 
Arends-Kuenning, M. y S. Amin. 2004. "School incentive programs and children's activities: The case of Bangladesh," Comparative Education Review 48(3): 295-317.

Biddlecom, A., R. Gregory, C.B. Lloyd, y B. Mensch. 2006. "Premarital sex and schooling transitions in four sub-Saharan African countries," Trabajo para el seminario de IUSSP sobre transiciones reproductivas y sexuales de adolescentes en países en desarrollo, 6 a 9 de noviembre, Cholula, México.

Brady, M., R. Assaad, B. Ibrahim, et al. 2007. "Providing New Opportunities to Adolescent Girls in Socially Conservative Settings: The Ishraq Program in Rural Upper Egypt-Full Report'. Nueva York: Population Council.

Ghuman, S. y C.B. Lloyd. 2007. "Teacher absence as a factor in gender inequalities in access to primary schooling in rural Pakistan," Poverty, Gender, and Youth Working Paper No.1. New York: Population Council.

Grant, M. y K. Hallman. 2006. "Pregnancy-related school dropout and prior school performance in South Africa," Policy Research Division Working Paper No. 212. Nueva York: Population Council.

Hallman, K.K. y S. Peracca con J. Catino y M. J. Ruiz. 2007. "A four-fold disadvantage: The effects of ethnicity, gender, poverty and geography on education in Guatemala," en M. Lewis y M. Lockheed (ed.), Exclusion, Gender and Education: Case Studies from the Developing World. Washington, DC: Center for Global Development.

Hallman, K.K. 2007. "Nonconsensual sex, school enrollment, and educational outcomes in South Africa," Africa Insight 37(3): 454-472.

Hewett P.C. y C.B. Lloyd. 2005. "Progress towards education for all: Trends and current challenges for sub-Saharan Africa," en C.B. Lloyd, J. Behrman, N.P. Stromquist y B. Cohen (eds.), The Changing Transitions to Adulthood in Developing Countries: Selected Studies. Washington, DC: National Academies Press, pp. 84-117.

Horizons Program. 2004. "Transitions to adulthood in the context of AIDS in South Africa: The impact of exposure to life skills education on adolescent knowledge, skills and behavior," USAID y Population Council.

Lloyd, C.B., C.E. Kaufman y P. Hewett. 2000. "The spread of primary schooling in sub-Saharan Africa: Implications for fertility change," Population and Development Review 26(3): 483-515.

Lloyd, C.B, B.S. Mensch y W.H. Clark. 2000. "The effects of primary school quality on school dropout among Kenyan girls and boys," Comparative Education Review 44(2): 113-147.

Lloyd, C.B., S. El-Tawila, W.H. Clark y B.S. Mensch. 2003. "The impact of educational quality on school exit in Egypt," Comparative Education Review 47(4): 444-467.

Lloyd, C.B., C. Mete y Z.A. Sathar. 2005. "The effect of gender differences in primary school access, type, and quality on the decision to enroll in rural Pakistan," Economic Development and Cultural Change 53(3): 685-710.

Lloyd, C.B. 2005. "Schooling," en C.B. Lloyd (ed.), Growing Up Global: The Changing Transitions to Adulthood in Developing Countries. Washington, DC: The National Academies Press, pp. 67-167.

Lloyd, C.B. 2005. "Schooling and adolescent reproductive behavior in developing countries," trabajo preparatorio para el informe del Proyecto Millennium de la ONU, Public Choices, Private Decisions: Sexual and Reproductive Health and the Millennium Development Goals.
Lloyd C.B. y M. Grant. 2005. "Growing up in contemporary Pakistan: The separate experiences of males and females," en C.B. Lloyd, J. Behrman, N.P. Stromquist y B. Cohen (ed.), The Changing Transitions to Adulthood in Developing Countries: Selected Studies. Washington, DC: National Academies Press, pp. 320-366.

Lloyd, C.B., C. Mete yM.J. Grant. 2006. The implications of changing educational and family circumstances for children's grade progression in rural Pakistan: 1997-2004," Policy Research Division Working Paper No. 209. Nueva York: Population Council. [no citado]

Lloyd, C.B., C. Mete y M. Grant. 2007. "Rural girls in Pakistan: Constraints of policy and culture," en M. Lewis y M. Lockheed (ed.), Exclusion, Gender and Schooling: Case Studies from the Developing World. Washington, DC: Center for Global Development.

Lloyd, C.B., M. Grant y A. Ritchie. 2008. "Gender differences in time use among adolescents in developing countries: Implications of rising school enrollment rates," Journal of Research on Adolescence 18(1): 99-120.

Lloyd, C.B. y B.S. Mensch. 2008. "Marriage and childbirth as factors in school exit: An analysis of DHS data from sub-Saharan Africa," Population Studies 62(1): $1-13$.

Lloyd, C.B. y J. Young. 2009. New Lessons: The Power of Educating Adolescent Girls. A Girls Count Report on Adolescent Girls. Nueva York: Population Council.

Mahmud, S. y S. Amin. 2006. "Girls' schooling and marriage in rural Bangladesh," en E. Hannum y B. Fuller (ed.), Research in Sociology of Education, Vol. 15. Elsevier, pp. 71-99.

Mensch, B.S., W.H. Clark, C.B. Lloyd y A.S. Erulkar. 2001. "Premarital sex, schoolgirl pregnancy, and school quality in rural Kenya," Studies in Family Planning 32(4):285-301.

Mensch, B.S., B. L. Ibrahim, S.M. Lee y O. El-Gibaly. 2003. "Gender-role attitudes among Egyptian adolescents," Studies in Family Planning 34(1):8-18.

Mensch, B. S., S. Singh y J.B. Casterline. 2005. "Trends in the timing of first marriage among men and women in the developing world", en C.B. Lloyd, J.R. Behrman, N.P. Stromquist y B. Cohen (ed.), The Changing Transitions to Adulthood in Developing Countries: Selected Studies. Washington, DC: National Academies Press, pp. 118-171.

Montgomery, M.R. y P.C. Hewett. 2006. "Poverty and children's schooling: A structural equations approach using MICS data", en A. Minujin, E. Delamonica y M. Komarecki (eds.), Human Rights and Social Policies for Children and Women: The Multiple Indicator Cluster Survey (MICS) in Practice. Nueva York: New School University.

Sathar, Z.A., C.B. Lloyd, C. Mete y M. ul Haque. 2003. "Schooling opportunities for girls as a stimulus for fertility change in rural Pakistan," Economic Development and Cultural Change 51(3):677-698.

UNESCO. 2007. "Education for all by 2015: Will we make it?," EFA Global Monitoring Report 2008. París: UNESCO.

Zibani, Nadia, Abeer Salem y Martha Brady. 2011. "Scaling up asset-building programs for marginalized adolescent girls in socially conservative settings: The Ishraq program in rural Upper Egypt." Transitions to Adulthood Brief no.12. Nueva York: Population Council.

\section{(2) Population Council}

El Population Council cambia la manera en la que el mundo piensa acerca de los problemas de salud y desarrollo. Buscamos entender las causas y las consecuencias de la desigualdad de género y las disparidades en las oportunidades que surgen durante la adolescencia. Brindamos las evidencias para desarrollar mejores programas y políticas prácticas que garanticen una transición exitosa y productiva hacia la edad adulta en los países en vías de desarrollo. www.popcouncil.org

() 2011 The Population Council, Inc.

4 - Visite www.popcouncil.org/publications/serialsbriefs/TABriefs.asp para obtener todos los resúmenes de la serie Promoting healthy, safe, and productive transitions to adulthood (Promoción de transiciones a la vida adulta sanas, seguras y productivas). 